\title{
Plasma Channel Formation in Inert Gases He and Ar by Low-energy Electron Beam
}

\author{
Ilya Zvigintsev, Vladimir Grigoriev, Irina Kolmakova \\ Institute of Cybernetics \\ Tomsk Polytechnic University \\ Tomsk, Russia
}

\begin{abstract}
To effective use of low-energy electron beams for technological purposes it is necessary to transport them to the target. For this a previously created plasma channel is used or the beam is injected in a neutral gas and creates independently the plasma channel. A longitudinal external magnetic field is often used for more stable beam movement. In this work, we consider the question of plasma channel formation by the lowenergy electron beam when a drift tube filled with argon or helium. A mathematical model of plasma channel formation is developed with the passage of the high-current electron beam in low-pressure inert gases in the presence or absence of the external magnetic field. This model is a system of nonlinear partial differential equations. Based on the proposed model are given preliminary numerical calculations of plasma channel parameters. The current neutralization of the low-energy electron beam in the low-pressure gas can be obtained.
\end{abstract}

Keywords-electron beam; argon; helium; plasma channel formation

\section{PRIMARY EQUATIONS}

The gas ionization in the drift space is due to the ionization of the gas atoms by fast beam electrons and by fields on the front edge of the beam. Plasma channel parameters changes due to gas pressure, geometry of the drift tube and beam parameters. This is an electron density, a plasma temperature and a plasma conductivity. Plasma current appears, current neutralization of the beam and conditions of the beam transportation are change. Plasma current emergence changes beam magnetic field. This current must be taken into account in the calculation of the vector potential.

Below we consider a model describing the beam transport in the gas at pressures $p<0.1$ Torr in full charge neutralization of the beam. To obtain the results until full charge neutralization an additional model of initial conditions used.

Let the electron beam with radius $r_{b}$ is injected in a plane $z$ $=0$ along the axis $z$ of the drift tube with radius $R_{c}$. The basic heterogeneity associated with the beam and plasma density distribution along the radius because passing time of the beam through the pipe drift is much smaller than the beam rise time. Fields of beam and plasma in the cylindrical coordinate system described by a nonlinear equation:

$$
\frac{1}{r} \frac{\partial}{\partial r}\left(r \frac{\partial A_{z}}{\partial r}\right)=-\frac{4 \pi}{c}\left(j_{b z}+j_{p z}\right)
$$

where $A_{z}$ is the vector potential, which satisfies the boundary conditions $A_{z}\left(r=R_{c}\right)=\partial A_{z} /\left.\partial r\right|_{r=0}=0$ and defines the electric field $E_{z}=-(1 / c)\left(\partial A_{z} / \partial t\right)$ and the magnetic field $B_{\theta}=-\partial A_{z} / \partial r$; $j_{b z}=e v_{b} n_{b}$ - the beam current density; $j_{p z}$ - the plasma current density; $c$ - the speed of light; $e$ is the elementary charge; $v_{b}$ is the speed of beam electrons; $n_{b}$ is the beam density. Let us suppose the drift tube has an ideal wall. The drift tube has ground potentials on its ends. The plasma current is accepted equal to zero in initial time. The initial condition for the vector potential is derived from (1).

An interaction between the plasma current density, the electric field and the plasma conductivity is defined by the equation:

$$
\frac{1}{v_{e f}} \frac{\partial j_{p z}}{\partial t}=\sigma E_{z}-j_{p z}
$$

where $\sigma=e^{2} n_{e} /\left(m_{e} v_{e f}\right)$ is the plasma conductivity; $m_{e}$ is electron mass; $n_{e}$ is the plasma electron density; $v_{e f}=v_{e a}+v_{e i}^{(1)}+v_{e i}^{(2)}$ is the effective collision frequency between plasma electrons and heavy particles; $v_{e a}$ is the frequency of collisions between plasma electrons and gas atoms; $\quad v_{e i}^{(l)}=1.45 \cdot 10^{-6} n_{i}^{(l)} T_{e}^{-3 / 2} \ln \left(2.4 \cdot 10^{20} T_{e}^{3} / n_{i}^{(l)}\right)$ is the frequency of collisions between plasma electrons and ions; $n_{i}{ }^{(l)}$ - the atomic $(l=1)$ and molecular $(l=2)$ ion density; $T_{e}$ is the electron plasma temperature, $\mathrm{eV}[1] ; v_{e a}=3.7 \cdot 10^{-8} n_{g} T_{e}$ for argon, $v_{e a}=4.4 \cdot 10^{-8} n_{g} T_{e}^{0.5}$ for helium [2]; $n_{g}$ is the gas density.

The balance of particles is described by the following equations:

$$
\begin{aligned}
& \frac{\partial n_{i}^{(1)}}{\partial t}=\left\langle\sigma_{i b} v_{b}\right\rangle n_{b} n_{g}+\left\langle\sigma_{i e} v_{e}\right\rangle n_{e} n_{g}-K_{k} n_{i}^{(1)} n_{g}^{2}- \\
& -\alpha_{r 1} n_{i}^{(1)} n_{e}^{2}-\alpha_{r 2} n_{i}^{(1)} n_{e} n_{g}-\alpha_{r 3} n_{i}^{(1)} n_{e}+\operatorname{div}\left(D_{A \perp} \operatorname{grad} n_{i}^{(1)}\right),
\end{aligned}
$$




$$
\frac{\partial n_{i}^{(2)}}{\partial t}=K_{k} n_{i}^{(1)} n_{g}^{2}-\alpha_{r d} n_{i}^{(2)} n_{e}
$$

$$
\begin{aligned}
& \frac{\partial n_{g}}{\partial t}=-\left\langle\sigma_{i b} v_{b}\right\rangle n_{b} n_{g}-\left\langle\sigma_{i e} v_{e}\right\rangle n_{e} n_{g}-K_{k} n_{i}^{(1)} n_{g}^{2}+ \\
& +\alpha_{r 1} n_{i}^{(1)} n_{e}^{2}+\alpha_{r 2} n_{i}^{(1)} n_{e} n_{g}+\alpha_{r 3} n_{i}^{(1)} n_{e}+2 \alpha_{r d} n_{i}^{(2)} n_{e},
\end{aligned}
$$

$$
n_{e}=n_{i}^{(1)}+n_{i}^{(2)}-n_{b},
$$

where $\sigma_{i b}, \sigma_{i e}$ are the ionization cross-sections by beam and plasma electrons [3]; $v_{e}$ is the speed of plasma electrons; $\alpha_{r 1}$, $\alpha_{r 2}, \alpha_{r 3}, \alpha_{r d}$ are the recombination coefficients: impact, with a neutral third particle, radiation and dissociative; $K_{k}$ is the conversion coefficient of atomic ions in molecular; $D_{A \perp}$ is the ambipolar diffusion coefficient. Boundary conditions for the plasma ion density and the ambipolar diffusion coefficient are similar to the vector potential boundary conditions. Recombination coefficients for argon atoms are determined by formulas:

$\alpha_{r 2}=1.09 \cdot 10^{-30} T_{e}^{-5 / 2} \mathrm{~cm}^{6} / \mathrm{s}$, $\alpha_{r 1}=4.7 \cdot 10^{-26} T_{e}^{-9 / 2} \mathrm{~cm}^{6} / \mathrm{s}$, $\alpha_{r d}=9.1 \cdot 10^{-7}\left(0.026 / T_{e}\right)^{0.61} \mathrm{~cm}^{3} / \mathrm{s}$. Recombination coefficients for helium atoms are determined by formulas: $\alpha_{r 1}=3.6 \cdot 10^{-27} T_{e}^{-9 / 2} \mathrm{~cm}^{6} / \mathrm{s}, \quad \alpha_{r 2}=1.07 \cdot 10^{-30} T_{e}^{-5 / 2} \mathrm{~cm}^{6} / \mathrm{s}$, $\alpha_{r 3}=6.45 \cdot 10^{-14} T_{e}^{-3 / 4} \mathrm{~cm}^{3} / \mathrm{s}, \quad \alpha_{r d}=7 \cdot 10^{-7}\left(0.026 / T_{e}\right)^{0.61} \mathrm{~cm}^{3} / \mathrm{s}$ $[4,5]$. Conversion coefficients: $K_{k}=2.5 \cdot 10^{-31} \mathrm{~cm}^{6} / \mathrm{s}$ for argon, $K_{k}=5.77 \cdot 10^{-32} \mathrm{~cm}^{6} / \mathrm{s}$ for helium [6]. The ambipolar diffusion coefficient is calculated according to the formula:

$$
D_{A \perp}=\frac{c^{2}}{\frac{\Omega_{e}^{2}+v_{e a}^{2}}{v_{e a}}+\frac{m_{i}}{m_{e}} \frac{4 \Omega_{i}^{2}+v_{i a}^{2}}{2 v_{i a}}} \frac{T_{e}+T_{i}}{E_{0}},
$$

where $T_{i}$ is plasma ion temperature, $\mathrm{eV} ; \Omega_{e}, \Omega_{i}$ is the cyclotron frequencies of the plasma electrons and ions; $m_{i}$ is the ion mass; $v_{i a}=4.6 \cdot 10^{-10} n_{g} T_{i}^{0.5}$ is the frequency of collisions between plasma ions and gas atoms [1].

An interaction between the plasma electron temperature and the electric field includes both elastic and not elastic collisions of the plasma electrons with heavy particles [7]:

$$
\frac{\partial T_{e}}{\partial t}=\frac{2}{3} \frac{E_{0} r_{0}}{m_{e} v_{e f}}\left(E_{z}^{2}-p^{2}\left(\frac{T_{e}}{k}\right)^{8}\right),
$$

where $k=11.65$ for argon and $k=18$ for helium [2]; $r_{0}$ is the classical electron radius; $E_{0}$ is the electron mass energy equivalent, $\mathrm{eV}$. The initial values of the plasma electron temperature take equal to $5 \mathrm{eV}$.

As the stated, the model describes beam transportation in the conditions of full charge neutralization, it is necessary to define initial conditions for plasma density at various beam injection modes.

\section{INITIAL CONDITIONS}

When the electron beam injection into a neutral gas, basic processes of the plasma channel formation are passing current ionization and plasma electron ionization in the space-charge field of the beam:

$$
\begin{gathered}
\frac{d n_{i}}{d t}=\sigma_{i b} n_{g} \frac{j_{q}}{e}+\sigma_{i e} n_{g} v_{e} n_{e}, \\
\frac{d n_{e}}{d t}=\frac{d n_{i}}{d t}-\frac{n_{e}}{\tau_{e}}, \\
n_{g}=3.5 \cdot 10^{16} p-n_{i},
\end{gathered}
$$

where $n_{i}$ is the plasma ion density; $j_{q}$ is the passing current density; $\tau_{e}=L / v_{e}$ is the constant of electron liberation time from the drift tube; $L$ is the length of the drift tube.

When the beam is injected in neutral gas, there is no compensation of a beam space charge. In a sufficiently strong external magnetic field $B_{z}$ the beam is kept from dispersion. The losses of the transported beam current associated with the formation of a virtual cathode when

$$
I_{b} \geq I_{p r}
$$

where $I_{b}$ is the injected beam current; $I_{p r}$ is the passing current. The virtual cathode (VC) arises at distance $d=1.5 \cdot 10^{-3} U^{3 / 4} / j_{b}{ }^{0.5}$ from the injection plane, where $j_{b}$ is the injected beam current density; $U-\mathrm{VC}$ voltage. Only part of injected beam current passes through the drift tube. It does not exceed the limiting current, which equals

$$
I_{p r}=I_{p r}^{(0)} /(1-f)
$$

$$
I_{p r}^{(0)}=\frac{2}{9} I_{A}^{(0)} \beta^{3}\left(1+\frac{4}{3} \ln \left(\frac{R_{c}}{r_{b}}\right)\right)^{-3 / 2}
$$

where $I_{p r}{ }^{(0)}$ is the vacuum limiting current [8], $f=n_{i} / n_{b}$ is the degree of charge neutralization of the beam; $I_{A}{ }^{(0)}$ is Alfven critical current; $\beta$ - the relative velocity of electron. The formula (14) gives tolerable results when $R_{c} / r_{b}$ range within 1 to 10 . VC disappears when (12) is false, and the beam current starts to pass fully. 
The passing beam electrons ionize gas. As a result, the degree of the beam charge neutralization increases with time. This leads to an increase of the passing current. The full charge neutralization is achieved at instant of time, when $f$ becomes equal to one. The values of the beam parameters and the plasma parameters at this time is taken as the initial conditions.

Here we describe the initial conditions in the presence of a strong external magnetic field. The above model initial conditions include a separate unit in the total ionization and plasma channel formation program. This model takes into account the transition mode. A similar model for the highcurrent beams in the absence of an external magnetic field is represented in the work [9].

\section{SiMULATED RESULTS}

Consider a uniform beam injection into the drift tube filled with inert gas. The beam parameters correspond to experiment [10]: $r_{b}=2 \mathrm{~cm}, R_{c}=4.1 \mathrm{~cm}, L=20 \mathrm{~cm}$. The beam profile [10] (current is $-300 \mathrm{~A}$, pulse duration is $145 \mu \mathrm{s}$, front edge duration is $20 \mu \mathrm{s}$ ) is shown in fig. 1a.

From the analysis of numerical calculations that the full charge neutralization in argon is achieved quickly enough. For helium it is achieved by the middle of the front edge of the beam. The plasma current responds to beam current variation slower at lower amplitude values of beam current than at high (fig. 1). When the pulse current equals $-300 \mathrm{~A}$ the plasma current amount to $75 \mathrm{~A}(25 \%)$. When the pulse current equals $-5 \mathrm{kA}$, the plasma current amount to $2.6 \mathrm{kA}(52 \%)$. The plasma current reached maximum values earlier than the beam current. On the shelf of the injected current pulse the plasma current is practically absent. This is because the pulse duration is sufficiently large and damping of the plasma current happening fast enough. At the trailing edge of the plasma current changes direction and increases the total current.

Fig. 2 shows leading edge erosion of the beam due to the formation of VC. Argon ionization in the absence of the external magnetic field is faster helium ionization in the external magnetic field. This results in more rapid achievement of full charge neutralization. At higher pressures virtual cathode will disappear earlier or general will not be formed.

Fig. 3 and fig. 4 show the graphs of 'boundary' values of system parameters, in which VC is not formed. The beam density becomes greater when the beam energy becomes less. The gas pressure must be increased to increase the ionization rate and reduce the time blurring $\mathrm{VC}$.

Thus, the model allows obtaining the electron density, which is very difficult to experimentally verify.

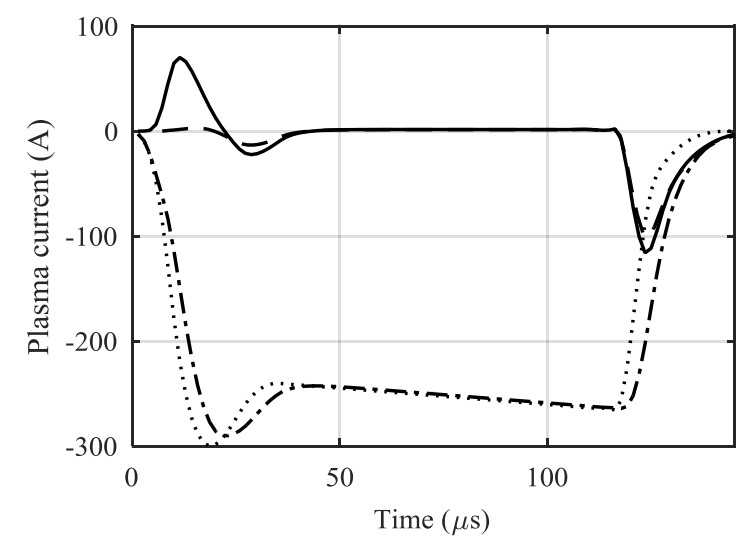

(a)

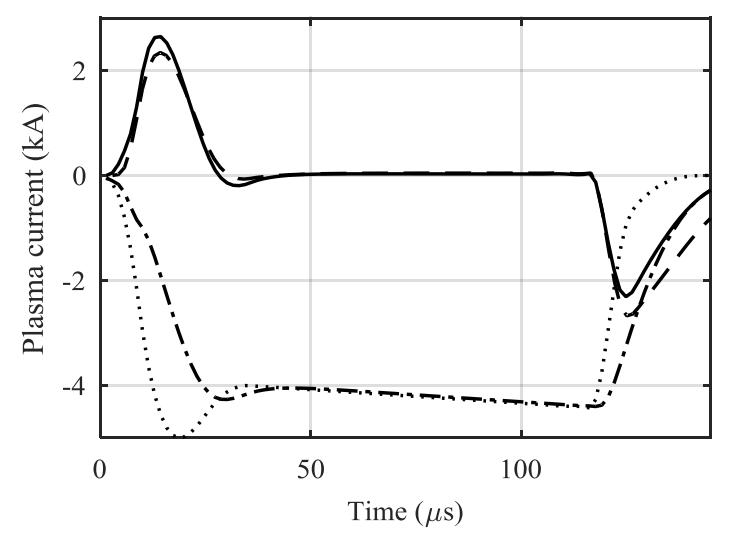

(b)

Fig. 1. The time dependences of the plasma current (solid line - Ar, dashed line $-\mathrm{He}$ ), the beam current (dotted line) and the total current (dash-dot line). $\varepsilon_{b}=10 \mathrm{keV}, B_{z}=1.5 \mathrm{kG}$. (a) $I_{b 0}=-300 \mathrm{~A}, p=2 \cdot 10^{-3}$ Torr. (b) $I_{b 0}=-5 \mathrm{kA}$, $p=5 \cdot 10^{-3}$ Torr

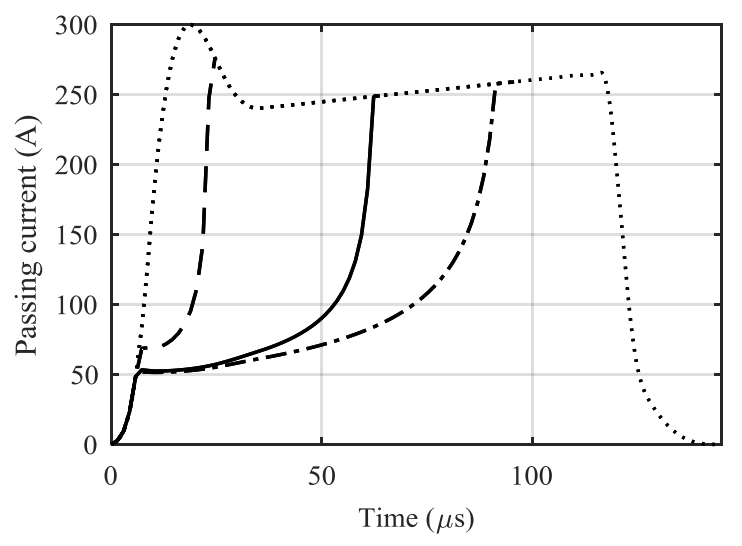

Fig. 2. The time dependence of the passing current. $p=1.5 \cdot 10^{-4}$ Torr, $\varepsilon_{b}=30 \mathrm{keV}, I_{b 0}=-300 \mathrm{~A}$. Solid line $-A r, B_{z}=0$; dashed line $-A r$ $B_{z}=1.5 \mathrm{kG}$; dash-dot line $-\mathrm{He}, B_{z}=1.5 \mathrm{kG}$; dotted line - the beam current. 


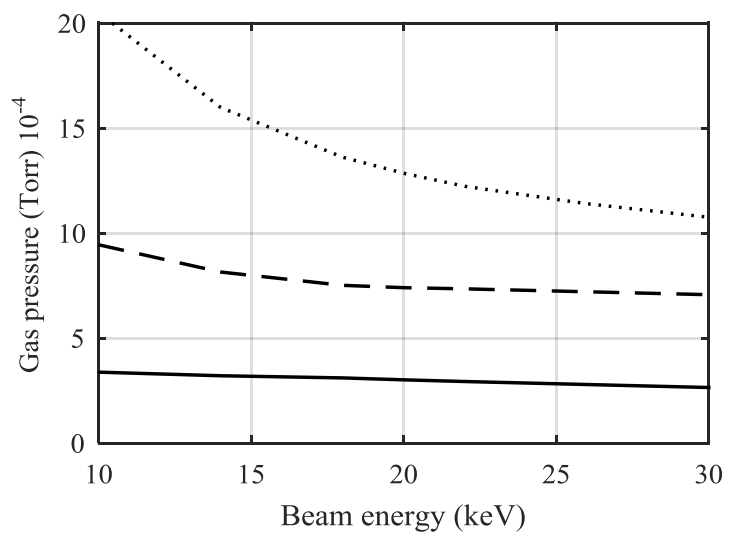

(a)

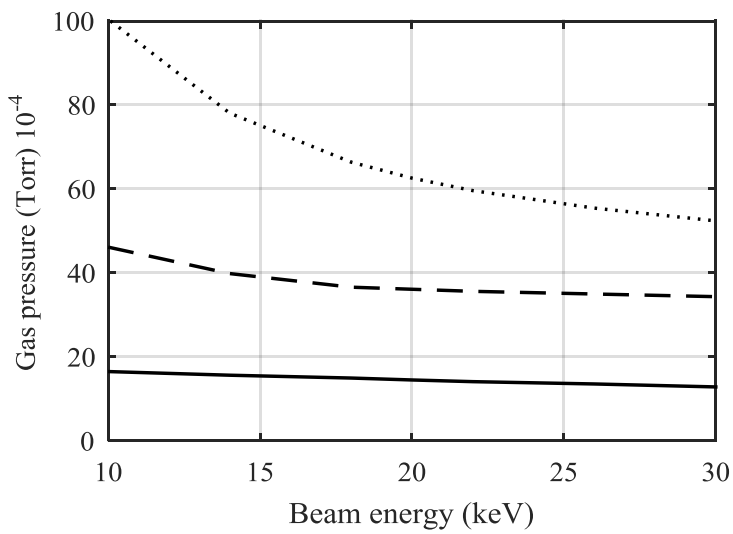

(b)

Fig. 3. 'Boundary' values of system parameters, in which virtual cathode is not formed, depending on the beam current, gas pressure and beam energy. Solid line $-I_{b 0}=-300 \mathrm{~A}$, dashed line $-I_{b 0}=-5 \mathrm{kA}$, dotted line $I_{b 0}=-15 \mathrm{kA}$. Argon (a), helium (b), $B_{z}=1.5 \mathrm{kG}$. VC is not formed when parameters are above curves. $\mathrm{VC}$ is formed when parameters are below curves.

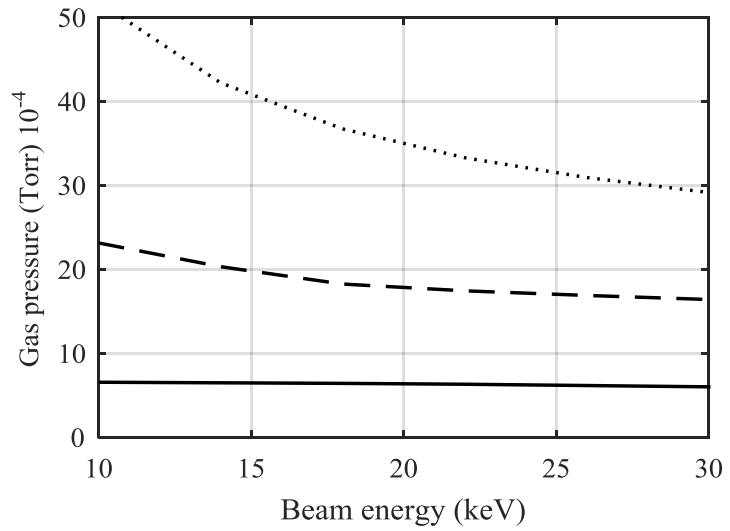

(a)

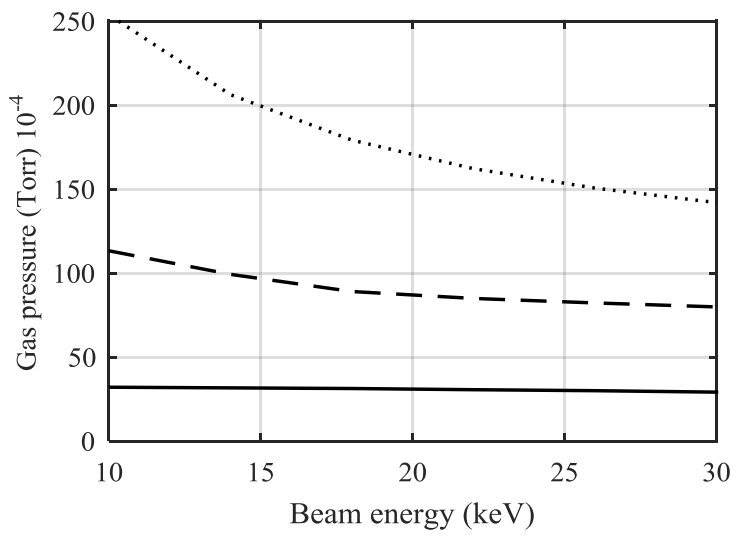

(b)

Fig. 4. 'Boundary' values of system parameters, in which virtual cathode is not formed, depending on the beam current, gas pressure and beam energy. Solid line $-I_{b 0}=-300 \mathrm{~A}$, dashed line $-I_{b 0}=-5 \mathrm{kA}$, dotted line $I_{b 0}=-15 \mathrm{kA}$. Argon (a), helium (b), $B_{z}=0 \mathrm{kG}$. VC is not formed when parameters are above curves. $\mathrm{VC}$ is formed when parameters are below curves

\section{REFERENCES}

[1] V.E. Golant, A.P. Zilinski and S.A Sakharov, "Fundamentals of plasma physics," Moscow: Atomizdat, 1977.

[2] A.V.Zakharov, "Investigation of the gas parameters influence on transportation of a high-intensity electron beam in the absence of external fields," Tomsk, 1987.

[3] M. Gryzinski, "Classical theory of atomic collisions. I. Theory of inelastic collisions," Physical Review, vol. 138, pp. 336-358, 1965.

[4] I. McDaniel and U. Nigan, "Gas lasers," Moscow: Mir, 1986.

[5] M. Mitchner and Ch. Kruger, "Partially ionized gases," Moscow: Mir, 1976.

[6] C. P. de Vriez and H. J. Oskam, "Four-body conversion of atomic helium ions," Phys. Rev. A22, 1429, 1980.

[7] V.P. Grigoriev and I.L. Zvigintsev, "Model of plasma channel formation by a low-energy electron beam in low pressure argon", Izvestia TPU, vol. 319, pp. 88-93, 2011

[8] A.N. Didenko, V.P. Grigoriev, and Y.P. Usov, "Power electron beam and their applications," Moscow: Atomizdat, 1977.

[9] I.L. Zvigintsev and V.P. Grigoriev, "Leading edge erosion of lowenergy electron beam during transport into neutral gas," Russian Physics Journal, vol. 55, pp. 460-462, 2012.

[10] S.V. Grigoryev, V.T. Astrelin, N.V. Devjatkov, I.V. Kandaurov, N.N. Koval, A.V. Kozyrev, P.V. Moskvin and A.D. Teresov, "Generation of submillisecond electron beam in the diode with the grid plasma cathode and the plasma anode generated by the asymmetrical reflective discharge," 16th International Symposium on High Current Electronics: Proceedings, pp. 19-22, 2010 